\title{
Remarkably Stable Chiral Zirconium Complexes for Asymmetric Mannich-type Reactions
}

\author{
Koichiro Saruhashi and Shū Kobayashi* \\ Graduate School of Pharmaceutical Sciences, The University of Tokyo, The HFRE \\ Division, ERATO, Japan Science Technology Agency (JST), Hongo, Bunkyo-ku, Tokyo \\ 113-0033, Japan
}

\section{Supporting Information}

\section{Experimental Section}

\section{General:}

${ }^{1} \mathrm{H}$ and ${ }^{13} \mathrm{C}$ NMR spectra were recorded on a JEOL ECX400, or ECX600 spectrometer in $\mathrm{CDCl}_{3}$, unless otherwise noted. Tetramethylsilane (TMS) served as internal standard (0 ppm) for ${ }^{1} \mathrm{H}$ NMR, and $\mathrm{CDCl}_{3}$ was used as internal standard (77.0 ppm) for ${ }^{13} \mathrm{C}$ NMR. XRF analyses was performed using Shimadzu EDX-800. HPLC was carried out using the following apparatuses; SHIMADZU LC-10AT (liquid chromatograph), SHIMADZU SPD-10A (UV detector), and SHIMADZU C-R6A Chromatopac. Column chromatography was conducted on Silica gel 60 (Merck) or Aluminium Oxide (activated, about 300 mesh, Wako), and preparative thin-layer chromatography was carried out using Wakogel B-5F. Commercially available chemicals and dry solvents (THF, $\mathrm{CH}_{2} \mathrm{Cl}_{2}, \mathrm{CH}_{3} \mathrm{CN}$, toluene, $\mathrm{CHCl}_{3}$ ), purchased from Aldrich, Kanto Chemical, Tokyo Chemical Industry and Wako Pure Chemical Industry, were purified according to standard procedures and dried over MS 4A. Zirconium tert-butoxide $\left(\mathrm{Zr}\left(\mathrm{O}^{t} \mathrm{Bu}\right)_{4}\right)$ and zirconium isopropoxide $\left(\mathrm{Zr}\left(\mathrm{O}^{i} \mathrm{Pr}\right)_{4}\right)$ were purchased from Tri Chemical Laboratory Inc. and Aldrich, respectively. Aldimines of aromatic and heteroaromatic aldehydes were prepared from the corresponding aldehydes and 2-aminophenol by usual method. ${ }^{1}$ The crude aldimines were recrystallized from ethanol to give the pure materials. Ketene silyl acetals were prepared according to the literature's methods. ${ }^{2}$ All reactions were carried out under argon atmosphere in well-dried glassware.

Preparation of powdered $\mathrm{Zr}$ catalysts. $\quad N$-Methylimidazole (NMI, $1.0 \mathrm{mmol}$ ) was added to a dichloromethane $(3.0 \mathrm{~mL})$ solution of a ligand $(1.0 \mathrm{mmol})$ and $\mathrm{Zr}(\mathrm{OR})_{4}(0.50$ 
mmol) at room temperature. After the mixture was stirred for $1 \mathrm{~h}$ at the same temperature, hexane $(100 \mathrm{~mL})$ was added and white precipitates were formed. The suspended mixture was further stirred overnight. Filtration of white suspension and washing with hexane afforded a $\mathrm{Zr}$ catalyst as white powder (Figure S-1).
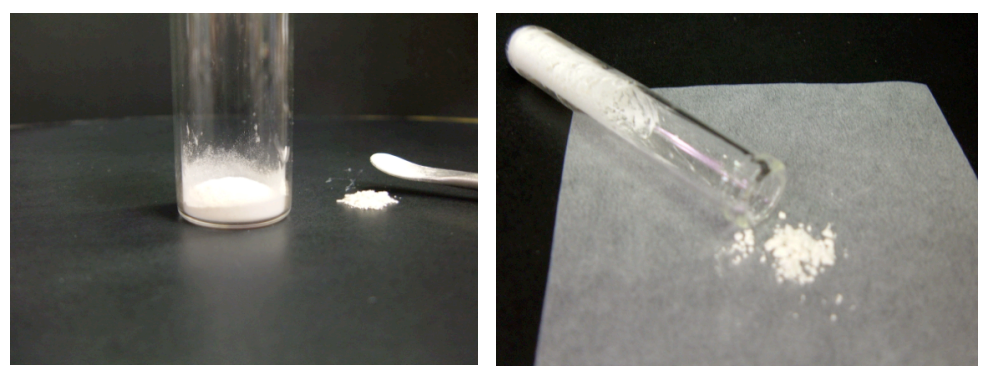

Figure S-1. Isolated chiral zirconium catalyst

Determination of the $\mathrm{Zr}$ contents in the powdered $\mathrm{Zr}$ catalysts. The powdered $\mathrm{Zr}$ catalyst $(15.0 \mathrm{mg})$ was placed in a $10 \mathrm{~mL}$-test tube, and sulfuric acid $(1.0 \mathrm{~mL})$ was added. The mixture was heated at $180{ }^{\circ} \mathrm{C}$ for $30 \mathrm{~min}$, and then nitric acid $(0.5 \mathrm{~mL})$ was added. The mixture was further heated for $1 \mathrm{~h}$ to give a clear solution. The solution was diluted with water, and the amount of the $\mathrm{Zr}$ metal was measured by XRF analysis (Table S-1).

Table S-1. Content of zirconium atom in the isolated catalysts

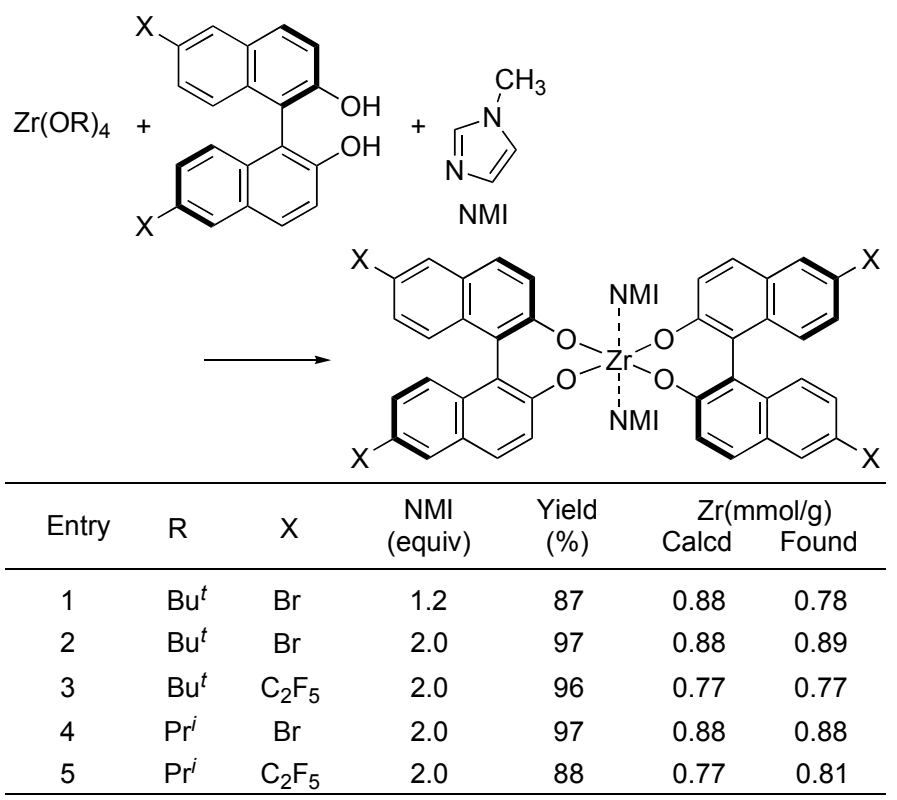


Typical experimental procedure for asymmetric Mannich-type reactions using a isolated powdered chiral zirconium catalyst. ${ }^{3}$

The powdered $\mathrm{Zr}$ catalyst $(0.040 \mathrm{mmol})$ was dissolved in dichloromethane $(1.00 \mathrm{~mL})$, and then an aldimine $(0.40 \mathrm{mmol})$ and a silicon enolate $(0.48 \mathrm{mmol})$ in dichloromethane $(0.75 \mathrm{~mL})$ were added at $-45^{\circ} \mathrm{C}$. The reaction mixture was stirred for $18 \mathrm{~h}$, and a saturated aqueous $\mathrm{NaHCO}_{3}$ solution was added to quench the reaction. After separation of the organic layer, the aqueous layer was extracted with $\mathrm{CH}_{2} \mathrm{Cl}_{2}$, and then the organic layers were combined and dried over anhydrous $\mathrm{Na}_{2} \mathrm{SO}_{4}$. After filtration and concentration under reduced pressure, the crude product obtained was treated with THF-1M HCl (10:1) at $0{ }^{\circ} \mathrm{C}$ for $1 \mathrm{~h}$. The solution was then basicified with a saturated aqueous $\mathrm{NaHCO}_{3}$ solution, and the aqueous solution was extracted with AcOEt. The combined organic layers were dried over anhydrous $\mathrm{Na}_{2} \mathrm{SO}_{4}$. After filtration and concentration under reduced pressure, the crude product was purified by preparative thin layer chromatography to afford the desired product. The optical purity was determined by HPLC analysis using a chiral column (vide infra).

\section{Methyl (2-hydroxyphenylamino)-2,2-dimethyl-3-phenylpropionate:}

${ }^{1} \mathrm{H}$ NMR $\left(\mathrm{CDCl}_{3}\right): \delta=1.21(\mathrm{~s}, 3 \mathrm{H}), 1.24(\mathrm{~s}, 3 \mathrm{H}), 3.68(\mathrm{~s}, 3 \mathrm{H}), 4.57(\mathrm{~s}, 1 \mathrm{H}), 6.36-6.76$ $(\mathrm{m}, 4 \mathrm{H}), 7.21-7.28(\mathrm{~m}, 5 \mathrm{H}) ;{ }^{13} \mathrm{C} \mathrm{NMR}\left(\mathrm{CDCl}_{3}\right): \delta=19.9,24.2,47.3,52.3,64.3,113.2$, 114.1, 117.6, 120.8, 127.3, 127.9, 128.3, 135.6, 138.9, 144.0, 178.0. HPLC (Daicel Chiralpak AD, hexane $/ \mathrm{PrOH} 9: 1$, flow rate $=1.0 \mathrm{~mL} / \mathrm{min}): t_{\mathrm{R}}=9.3 \mathrm{~min}(R), t_{\mathrm{R}}=16.0$ $\min (S)$.

\section{Methyl (2-hydroxyphenylamino)-2,2-dimethyl-3-naphthylpropionate:}

${ }^{1} \mathrm{H}$ NMR $\left(\mathrm{CDCl}_{3}\right): \delta=1.18(\mathrm{~s}, 3 \mathrm{H}), 1.25(\mathrm{~s}, 3 \mathrm{H}), 3.66(\mathrm{~s}, 3 \mathrm{H}), 5.62(\mathrm{~s}, 1 \mathrm{H}), 6.28-6.62$ $(\mathrm{m}, 4 \mathrm{H}), 7.22-8.00(\mathrm{~m}, 7 \mathrm{H}) ;{ }^{13} \mathrm{C} \mathrm{NMR}\left(\mathrm{CDCl}_{3}\right): \delta=19.9,25.1,48.4,52.4,57.8,113.4$, 114.2, 117.9, 121.2, 122.1, 123.2, 125.2, 125.3, 125.4, 126.1, 128.1, 129.1, 133.6, 135.3, 135.5, 144.1, 177.9. HPLC (Daicel Chiralcel OD-H, hexane $/{ }^{i} \operatorname{PrOH} 19: 1$, flow rate $=$ $0.5 \mathrm{~mL} / \mathrm{min}): t_{\mathrm{R}}=20.4 \mathrm{~min}(S), t_{\mathrm{R}}=24.4 \mathrm{~min}(R)$.

\section{Methyl 3-(4-chlorophenyl)-3-(2-hydroxyphenylamino)-2,2-dimethylpropionate:}

${ }^{1} \mathrm{H}$ NMR $\left(\mathrm{CDCl}_{3}\right): \delta=1.19$ (s, 3H), 1.24 (s, 3H), 3.67 (s, 3H), 4.55 (s, 1H), 6.31-6.90 $(\mathrm{m}, 4 \mathrm{H}), 7.22(\mathrm{~s}, 2 \mathrm{H}), 7.35(\mathrm{~s}, 2 \mathrm{H}) ;{ }^{13} \mathrm{C} \mathrm{NMR}\left(\mathrm{CDCl}_{3}\right): \delta=20.2,24.7,47.3,52.4,64.0$, $113.3,114.3,117.9,121.1,128.2,128.3,129.7,133.2,135.4,137.7,144.0,177.5$. HPLC (Daicel Chiralpak AD, hexane $/{ }^{i} \mathrm{PrOH} 9: 1$, flow rate $=1.0 \mathrm{~mL} / \mathrm{min}$ ): $t_{\mathrm{R}}=8.3 \mathrm{~min}$ $(R), t_{\mathrm{R}}=16.7 \min (S)$. 


\section{S-Ethyl 3-(2-hydroxyphenylamino)- 3-phenylpropanethioate:}

${ }^{1} \mathrm{H} \mathrm{NMR}\left(\mathrm{CDCl}_{3}\right): \delta=1.67(\mathrm{t}, J=7.3 \mathrm{~Hz}, 3 \mathrm{H}), 2.83(\mathrm{q}, J=7.3 \mathrm{~Hz}, 2 \mathrm{H}), 2.97(\mathrm{dd}, J=$ 5.4, $14.9 \mathrm{~Hz}, 1 \mathrm{H}), 3.07$ (dd, $J=8.1,14.9 \mathrm{~Hz}, 1 \mathrm{H}), 4.81$ (dd, $J=5.4,14.9 \mathrm{~Hz}, 1 \mathrm{H})$, 6.44-6.71 (m, 4H), 7.20-7.33 (m, 5H); ${ }^{13} \mathrm{C} \mathrm{NMR}\left(\mathrm{CDCl}_{3}\right): \delta=14.4,23.6,51.4,56.1$, $114.4,114.6,118.8,121.1,126.3,127.4,128.6,134.9,141.7,144.7,198.4$. HPLC (Daicel Chiralpak AS, hexane $/{ }^{i} \mathrm{PrOH} 19: 1$, flow rate $\left.=1.0 \mathrm{~mL} / \mathrm{min}\right): t_{\mathrm{R}}=26.6 \mathrm{~min}(S)$, $t_{\mathrm{R}}=38.2 \min (R)$.

\section{S-Ethyl 3-(2-hydroxyphenylamino)- 3-naphthylpropanethioate:}

${ }^{1} \mathrm{H}$ NMR $\left(\mathrm{CDCl}_{3}\right): \delta=1.18(\mathrm{t}, J=7.3 \mathrm{~Hz}, 3 \mathrm{H}), 2.86(\mathrm{q}, J=7.3 \mathrm{~Hz}, 2 \mathrm{H}), 3.03(\mathrm{dd}, J=$ 9.4, $14.8 \mathrm{~Hz}, 1 \mathrm{H}), 3.20(\mathrm{dd}, J=3.3,14.8 \mathrm{~Hz}, 1 \mathrm{H}), 5.68-5.70(\mathrm{~m}, 1 \mathrm{H}), 6.30-6.71(\mathrm{~m}, 4 \mathrm{H})$, 7.29-8.23 (m, 7H); ${ }^{13} \mathrm{C} \mathrm{NMR}\left(\mathrm{CDCl}_{3}\right): \delta=14.4,23.7,50.7,51.9,113.5,114.4,118.1$, 121.3, 122.1, 123.1, 125.6, 125.7, 126.5, 128.0, 129.2, 130.3, 134.0, 135.3, 136.7, 143.9, 198.5. HPLC (Daicel Chiralpak AD, hexane $/{ }^{i} \mathrm{PrOH} 9: 1$, flow rate $=1.0 \mathrm{~mL} / \mathrm{min}$ ): $t_{\mathrm{R}}=$ $15.5 \min (S), t_{\mathrm{R}}=21.0 \min (R)$.

\section{S-Ethyl 3-(4-chlorophenyl)-3-(2-hydroxyphenylamino)-propanethioate:}

${ }^{1} \mathrm{H} \mathrm{NMR}\left(\mathrm{CDCl}_{3}\right): \delta=1.21(\mathrm{t}, J=7.4 \mathrm{~Hz}, 3 \mathrm{H}), 2.87(\mathrm{q}, J=7.4 \mathrm{~Hz}, 2 \mathrm{H}), 2.96(\mathrm{dd}, J=$ $5.1,8.3 \mathrm{~Hz}, 1 \mathrm{H}), 3.05(\mathrm{dd}, J=8.3,14.9 \mathrm{~Hz}, 1 \mathrm{H}), 4.78(\mathrm{dd}, J=5.1,8.3 \mathrm{~Hz}, 1 \mathrm{H})$, 6.39-6.78 (m, 4H), 7.22-7.28 (m, 4H); ${ }^{13} \mathrm{C} \mathrm{NMR}\left(\mathrm{CDCl}_{3}\right): \delta=14.5,23.7,51.2,55.6$, $114.5,115.0,119.3,121.2,127.8,128.9,133.2,134.6,140.3,144.7,197.8$. HPLC (Daicel Chiralpak AD, hexane $/{ }^{i} \mathrm{PrOH} 9: 1$, flow rate $\left.=1.0 \mathrm{~mL} / \mathrm{min}\right): t_{\mathrm{R}}=19.5 \mathrm{~min}(S), t_{\mathrm{R}}$ $=24.3 \min (R)$.

\section{S-Ethyl 3-[((2-hydroxy-6-methylphenyl)amino)-5-methylhexane-thioate:}

${ }^{1} \mathrm{H}$ NMR $\left(\mathrm{CDCl}_{3}\right)$; anti: $\delta=0.81(\mathrm{~d}, J=6.4 \mathrm{~Hz}, 3 \mathrm{H}), 0.88(\mathrm{~d}, J=6.6 \mathrm{~Hz}, 3 \mathrm{H}), 1.26(\mathrm{t}, J$ $=7.4,3 \mathrm{H}), 1.35(\mathrm{dd}, J=6.6,7.7 \mathrm{~Hz}, 2 \mathrm{H}), 1.63(\mathrm{~d}, J=6.7 \mathrm{~Hz}, 1 \mathrm{H}), 2.25(\mathrm{~s}, 3 \mathrm{H}), 2.64$ $(\mathrm{dd}, J=8.0,15.9 \mathrm{~Hz}, 1 \mathrm{H}), 2.80(\mathrm{dd}, J=4.2,15.8,1 \mathrm{H}), 2.92(\mathrm{q}, J=7.5 \mathrm{~Hz}, 2 \mathrm{H}), 3.62$ $(\mathrm{dq}, J=4.2,7.1 \mathrm{~Hz}, 1 \mathrm{H}), 6.66(\mathrm{dd}, J=0.6,7.4,1 \mathrm{H}), 6.76(\mathrm{dd}, J=1.4,7.8 \mathrm{~Hz}, 2 \mathrm{H}), 6.88$ $(\mathrm{t}, J=7.7 \mathrm{~Hz}, 1 \mathrm{H}) ;{ }^{13} \mathrm{C} \mathrm{NMR}\left(\mathrm{CDCl}_{3}\right): \delta=14.6,18.1,22.0,23.2,23.6,25.1,44.5,48.4$, 52.5, 113.3, 121.8, 124.6, 131.2, 132.5, 151.9, 200.3. HPLC (Daicel Chiralpak OD, hexane $/{ }^{i} \mathrm{PrOH} 60: 1$, flow rate $\left.=1.0 \mathrm{~mL} / \mathrm{min}\right): t_{\mathrm{R}}=14.9 \mathrm{~min}(S), t_{\mathrm{R}}=17.2 \mathrm{~min}(R)$.

\section{3-(2-hydroxyphenyl)amino-2-methyl-3-phenylpropanoate:}

${ }^{1} \mathrm{H} \mathrm{NMR}\left(\mathrm{CDCl}_{3}\right)$ : anti: $\delta=1.24(\mathrm{~d}, J=7.0 \mathrm{~Hz}, 3 \mathrm{H}), 3.12(\mathrm{dq}, J=7.0,8.7 \mathrm{~Hz}, 1 \mathrm{H})$, 
$4.55(\mathrm{~d}, J=8.7 \mathrm{~Hz}, 1 \mathrm{H}), 6.44-6.69(\mathrm{~m}, 4 \mathrm{H}), 6.98-7.00(\mathrm{~m}, 2 \mathrm{H}), 7.13-7.44(\mathrm{~m}, 8 \mathrm{H})$; syn: $\delta=1.36(\mathrm{~d}, J=7.1 \mathrm{~Hz}, 3 \mathrm{H}), 3.24(\mathrm{dq}, J=6.0,7.1 \mathrm{~Hz}, 1 \mathrm{H}), 4.89(\mathrm{~d}, J=6.0 \mathrm{~Hz}, 1 \mathrm{H})$, 6.44-6.69 (m, $4 \mathrm{H}), 6.81-6.83(\mathrm{~m}, 2 \mathrm{H}), 7.13-7.44(\mathrm{~m}, 8 \mathrm{H}) ;{ }^{13} \mathrm{C} \mathrm{NMR}\left(\mathrm{CDCl}_{3}\right)$ : anti: $\delta=$ 15.2 , 47.0, 61.7, 114.5, 114.9, 118.8, 120.9, 121.5, 126.0, 127.1, 127.7, 128.6, 129.4, $135.0,140.6,144.9,150.5,174.6$; syn: $\delta=12.4,46.7,60.7,113.2,114.3,117.8,121.2$, $121.4,125.9,127.1,127.5,128.6,129.3,135.7,140.6,143.7,150.4,173.2$. HPLC (Daicel Chiralcel OD-H, hexane $/{ }^{i} \mathrm{PrOH} 19: 1$, flow rate $=0.75 \mathrm{~mL} / \mathrm{min}$ ): anti: $t_{\mathrm{R}}=18.0$ $\min (\operatorname{minor}=2 S, 3 R), t_{\mathrm{R}}=35.4 \min ($ major $=2 R, 3 S)$; syn: $t_{\mathrm{R}}=23.3 \min (\operatorname{minor}=2 R$, $3 R), t_{\mathrm{R}}=44.9 \min ($ major $=2 S, 3 S)$;

\section{Phenyl 2,5-dimethyl-3-(2-hydroxy-6-methylphenyl)aminohexanoate:}

${ }^{1} \mathrm{H}$ NMR $\left(\mathrm{CDCl}_{3}\right)$ : anti: $\delta=0.85(\mathrm{~d}, J=6.6 \mathrm{~Hz}, 3 \mathrm{H}), 0.87(\mathrm{~d}, J=6.3 \mathrm{~Hz}, 3 \mathrm{H}), 1.38(\mathrm{~d}$, $J=7.1 \mathrm{~Hz}, 3 \mathrm{H}), 1.40-1.49(\mathrm{~m}, 1 \mathrm{H}), 1.62-1.76(\mathrm{~m}, 1 \mathrm{H}), 2.29(\mathrm{~s}, 3 \mathrm{H}), 2.85(\mathrm{dq},=5.7$, $7.1 \mathrm{~Hz}, 1 \mathrm{H}), 3.77(\mathrm{dt}, J=5.7,7.3 \mathrm{~Hz}, 1 \mathrm{H}), 6.66-7.39(\mathrm{~m}, 8 \mathrm{H}) ; \boldsymbol{s y n}: \delta=0.88(\mathrm{~d}, J=6.3$ $\mathrm{Hz}, 3 \mathrm{H}), 0.92(\mathrm{~d}, J=6.6 \mathrm{~Hz}, 3 \mathrm{H}), 1.34(\mathrm{~d}, J=7.3 \mathrm{~Hz}, 3 \mathrm{H}), 1.40-1.49(\mathrm{~m}, 1 \mathrm{H})$, $1.62-1.76(\mathrm{~m}, 1 \mathrm{H}), 2.28(\mathrm{~s}, 3 \mathrm{H}), 2.93(\mathrm{dq}, J=2.8,7.3 \mathrm{~Hz}, 1 \mathrm{H}), 3.69(\mathrm{dt}, J=2.8,6.8 \mathrm{~Hz}$, $1 \mathrm{H}), 6.66-7.39(\mathrm{~m}, 8 \mathrm{H}) ;{ }^{13} \mathrm{C} \mathrm{NMR}\left(\mathrm{CDCl}_{3}\right)$ : anti: $\delta=13.2,18.4,22.4,23.2,25.1,43.3$, 44.2, 56.0, 113.4, 121.4, 122.5, 123.1, 125.9, 129.4, 130.7, 132.5, 149.8, 150.5, 175.0; syn: $\delta=10.9,18.2,22.5,22.9,25.1,41.1,41.8,56.1,113.6,121.4,122.2,124.1,126.0$, 129.4, 131.4, 132.4, 150.5, 151.4, 174.7; HPLC (Daicel Chiralpak AD (double), hexane $/{ }^{i} \mathrm{PrOH} 9: 1$, flow rate $\left.=0.80 \mathrm{~mL} / \mathrm{min}\right)$ :anti: $t_{\mathrm{R}}=17.9 \mathrm{~min}(\mathrm{~min}=2 S, 3 S), t_{\mathrm{R}}=$ $19.2 \min ($ major $=2 R, 3 R)$; syn: $t_{\mathrm{R}}=20.5 \min ($ major $=2 S, 3 R), t_{\mathrm{R}}=31.8 \mathrm{~min}($ minor $=$ $2 R, 3 S)$;

\section{Phenyl 2-benzyloxy-3-(2-hydroxyphenyl)amino-3-phenylpropionate:}

${ }^{1} \mathrm{H}$ NMR anti: $\delta 4.51(\mathrm{~d}, 1 \mathrm{H}, J=11.7 \mathrm{~Hz}), 4.56(\mathrm{~d}, 1 \mathrm{H}, J=5.4 \mathrm{~Hz}), 4.78(\mathrm{~d}, 1 \mathrm{H}, J=$ $11.7 \mathrm{~Hz}), 4.95(\mathrm{~d}, 1 \mathrm{H}, J=5.4 \mathrm{~Hz}), 6.51-6.73(\mathrm{~m}, 4 \mathrm{H}), 6.80-6.92(\mathrm{~m}, 2 \mathrm{H}), 7.12-7.20(\mathrm{~m}$, 2H), 7.24-7.28 (m, 6H), 7.33 (s, 3H), 7.43-7.45 (m, 2H). syn: $\delta 4.45$ (d, 1H, $J=12.1$ $\mathrm{Hz}), 4.74(\mathrm{~d}, 1 \mathrm{H}, J=3.9 \mathrm{~Hz}), 4.80(\mathrm{~d}, 1 \mathrm{H}, J=12.1 \mathrm{~Hz}), 5.08$ (brd, $1 \mathrm{H}, J=3.9 \mathrm{~Hz})$, 6.51-6.73 (m, 4H), 6.80-6.92 (m, 2H), 7.12-7.20 (m, 2H), 7.24-7.28 (m, 6H), $7.33(\mathrm{~s}$, 3H), 7.43-7.45 (m, 2H). 13C NMR anti: $\delta$ 59.8, 73.2, 80.6, 114.1, 114.5, 118.7, 119.0, $121.1,121.2$, 121.2, 126.0, 127.9, 128.1, 128.3, 128.4, 128.4, 129.3, 136.7, 138.3, 144.4, 150.0, 169.6. syn: $\delta$ 60.7, 73.0, 79.6, 113.4, 115.3, 117.7, 118.4, 120.4, 121.1, 121.3, 125.9 , 128.0, 128.1, 128.2, 128.3, 128.5, 129.2, 136.4, 136.5, 144.3, 149.8, 169.5 . HPLC: Daicel Chiralcel OD, hexane $/ \mathrm{PrOH}=19 / 1$, flow rate $=1.0 \mathrm{ml} / \mathrm{min}: t_{\mathrm{R}}=20.0$ $\min (2 R, 3 S), t_{\mathrm{R}}=24.5 \min (2 S, 3 R), t_{\mathrm{R}}=29.5 \min (2 S, 3 S), t_{\mathrm{R}}=32.1 \mathrm{~min}(2 R, 3 R)$. 


\section{Isopropyl 2-tert-butyldimethylsiloxy-3-(2-methoxyphenyl)amino-3-phenyl- propionate}

${ }^{1} \mathrm{H}$ NMR syn: $\delta 0.00(\mathrm{~s}, 6 \mathrm{H}), 0.93(\mathrm{~s}, 9 \mathrm{H}), 1.23(\mathrm{~d}, 3 \mathrm{H}, J=6.3 \mathrm{~Hz}), 1.30(\mathrm{~d}, 3 \mathrm{H}, J=6.3$ $\mathrm{Hz}), 3.97(\mathrm{~s}, 3 \mathrm{H}), 4.48(\mathrm{~d}, 1 \mathrm{~h}, J=3.0 \mathrm{~Hz}), 4.97$ (dd, $1 \mathrm{H}, J=3.0,7.6 \mathrm{~Hz}), 5.11(\mathrm{dq}, 1 \mathrm{H}, J$ $=6.3 \mathrm{~Hz}), 5.53(\mathrm{~d}, 1 \mathrm{H}, J=7.6 \mathrm{~Hz}), 6.38(\mathrm{dd}, 1 \mathrm{H}, J=1.7,7.6 \mathrm{~Hz}), 6.54-6.85(\mathrm{~m}, 3 \mathrm{H})$, 7.26-7.46 (m, 5H). anti: $\delta=0.03(\mathrm{~s}, 3 \mathrm{H}), 0.06(\mathrm{~s}, 3 \mathrm{H}), 1.00(\mathrm{~s}, 9 \mathrm{H}), 1.13(\mathrm{~d}, 3 \mathrm{H}, J=6.3$ $\mathrm{Hz}), 1.26(\mathrm{~d}, 3 \mathrm{H}, J=6.3 \mathrm{~Hz}), 3.93(\mathrm{~s}, 3 \mathrm{H}), 4.61$ (d, $1 \mathrm{H}, J=5.3 \mathrm{~Hz}), 4.83$ (dd, 1H, $J=$ $5.3,8.3 \mathrm{~Hz}), 5.00(\mathrm{dq}, 1 \mathrm{H}, J=6.3 \mathrm{~Hz}), 5.38(\mathrm{~d}, 1 \mathrm{H}, J=8.3 \mathrm{~Hz}), 6.54-6.85(\mathrm{~m}, 4 \mathrm{H})$, 7.26-7.46 (m, 5H). 13C NMR syn: $\delta$-5.9, -5.3, 18.1, 21.6, 21.7, 25.5, 55.5, 60.2, 68.6, $76.8,109.5,111.1,116.2,121.1,127.2,127.9,128.2,136.8,140.0,146.9,171.2$. anti: $\delta$ $=-5.9,-5.2,18.2,21.5,21.6,25.6,55.2,59.8,55.2,59.8,68.5,75.4,109.3,111.1,116.6$, 121.1, 127.5, 127.7, 128.5, 136.3, 138.1, 147.0, 171.1. HPLC: Daicel CHIRALPAK AD x 2 ; hexane $/{ }^{i} \mathrm{PrOH}=400 / 1$; flow $0.8 \mathrm{ml} / \mathrm{min}: t_{\mathrm{R}}=21.7(2 S, 3 R), t_{\mathrm{R}}=25.7(2 R$, $3 S)$.

Procedure for the reuse of the isolated catalysts. The powdered $\mathrm{Zr}$ catalyst $(0.040$ mmol) was dissolved in dichloromethane $(1.00 \mathrm{~mL})$. To the solution was added an aldimine $(0.40 \mathrm{mmol})$ and a silicon enolate $(0.48 \mathrm{mmol})$ in dichloromethane $(0.75 \mathrm{~mL})$ at $-45^{\circ} \mathrm{C}$. The reaction mixture was stirred for $18 \mathrm{~h}$, and hexane $(10 \mathrm{~mL})$ was added to quench the reaction. Precipitates were formed, and the suspended mixture was wormed to room temperature followed by addition of hexane $(50 \mathrm{~mL})$. By filtration of the hexane suspension, the $\mathrm{Zr}$ catalyst was recovered as white powder ( $>95 \%)$.

Preparation of the single crystals. (a) The powdered $\mathrm{Zr}$ catalyst containing $\mathrm{N}$-benzylimidazole $(20 \mathrm{mg})$ was dissolved in toluene $(2 \mathrm{~mL})$. After standing the solution in dry argon atmosphere for 1 day, colorless cubic single crystals were formed (Figure S-2). (b) The powdered Zr catalyst containing $N$-benzylimidazole (20 mg) was dissolved in AcOEt $(2 \mathrm{~mL})$. This solution was stood in a sealed vial filled with hexane. After standing the solution for $8 \mathrm{~h}$, colorless single crystals were formed. 

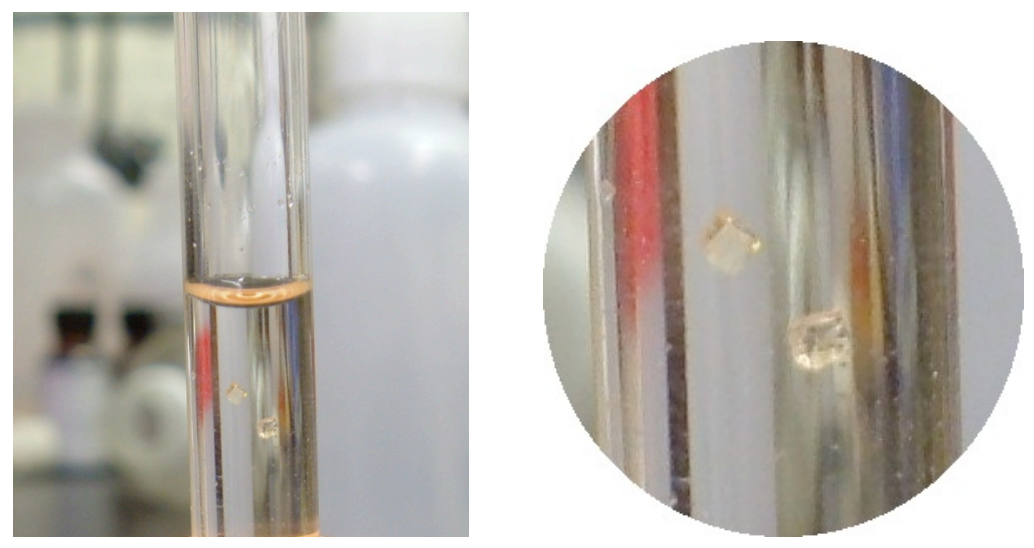

Figure S-2. Single crystals of the powdered $\mathrm{Zr}$ catalyst in toluene.

Procedure for asymmetric Mannich-type reactions using single crystals (Scheme S-1). Without NMI: The crystal Zr catalyst (31 mg, $0.010 \mathrm{mmol}$ ) was suspended in dichloromethane $(0.5 \mathrm{~mL})$. After the mixture was stirred for $1 \mathrm{~h}$ at room temperature, an aldimine $(0.20 \mathrm{mmol})$ and a silicon enolate $(0.24 \mathrm{mmol})$ in dichloromethane $(0.50$ $\mathrm{mL}$ ) were added at $-45^{\circ} \mathrm{C}$. The reaction mixture was stirred for $24-48 \mathrm{~h}$, and hexane $(10 \mathrm{~mL})$ was added to quench the reaction. Precipitates were formed, and the filtrate was concentrated and treated with THF-1M HCl (10:1) at $0{ }^{\circ} \mathrm{C}$ for $1 \mathrm{~h}$. The solution was then basicified with a saturated aqueous $\mathrm{NaHCO}_{3}$ solution, and the aqueous solution wasextracted with AcOEt. The organic layer was dried over anhydrous $\mathrm{Na}_{2} \mathrm{SO}_{4}$. After filtration and concentration under reduced pressure, the crude product was purified by preparative thin layer chromatography to afford the desired product.

With NMI: $N$-Methylimidazole $(6.4 \mu \mathrm{L}, 0.080 \mathrm{mmol})$ was added to white suspension in dichloromethane containing crystals of the $\mathrm{Zr}$ catalyst $(31 \mathrm{mg}, 0.010 \mathrm{mmol}$ ). After stirring for $3 \mathrm{~h}$, crystals were partially dissolved, and the slightly suspended mixture was used for the reaction. 

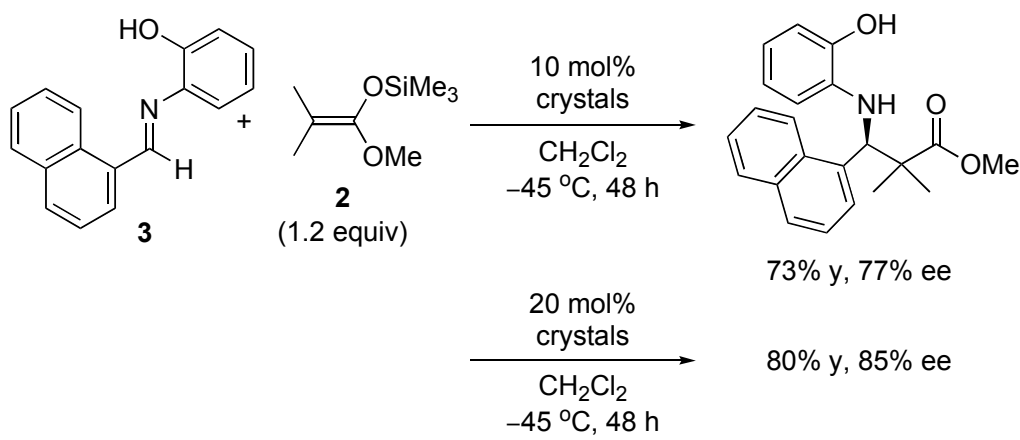

$73 \%$ y, $77 \%$ ee

$80 \%$ y, $85 \%$ ee
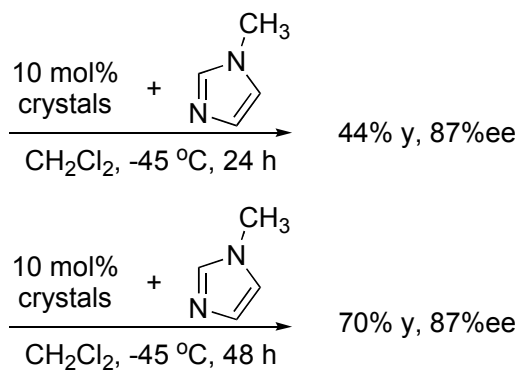

Scheme S-1. Asymmetric Mannich reactions using $\mathrm{Zr}$ single crystals

NMR Experiment. Catalyst Only (Figure S-3). A typical procedure is described for the preparation of the catalysts: (a) To 6,6'-dibromoBINOL $(0.08 \mathrm{mmol})$ and $\mathrm{Zr}\left(\mathrm{O}^{\mathrm{i}} \mathrm{Pr}\right)_{4}(0.04 \mathrm{mmol})$ in dichloromethane- $d^{2}(0.50 \mathrm{~mL})$ were added $N$-methylimidazole (0.48 mmol) at room temperature. The mixture was stirred for $1 \mathrm{~h}$ at the same temperature. (b) The catalyst $(20 \mathrm{mg})$ was dissolved in dichloromethane- $d^{2}(0.5 \mathrm{~mL})$. (c) Addition of dichloromethane- $d^{2}(0.5 \mathrm{~mL})$ to single crystals $(10 \mathrm{mg})$ afforded slightly suspended mixture. ${ }^{1} \mathrm{H}$ and ${ }^{13} \mathrm{C}$ NMR experiments of white suspension were performed. (d) $N$-Methylimidazole $(2.1 \mu \mathrm{L}, 8$ equiv to $\mathrm{Zr}$ ) was added to white suspension in dichloromethane- $d^{2}$ containing crystals $(10 \mathrm{mg})$. After stirred for $3 \mathrm{~h}$, crystals were completely dissolved and the suspension was changed to a colorless solution; and then ${ }^{1} \mathrm{H}$ and ${ }^{13} \mathrm{C}$ NMR experiments were performed. 
Figure S-3. ${ }^{1} \mathrm{H}$ and ${ }^{13} \mathrm{C}$ NMR spectra of $\mathrm{Zr}$ catalysts in $\mathrm{CD}_{2} \mathrm{Cl}_{2}$
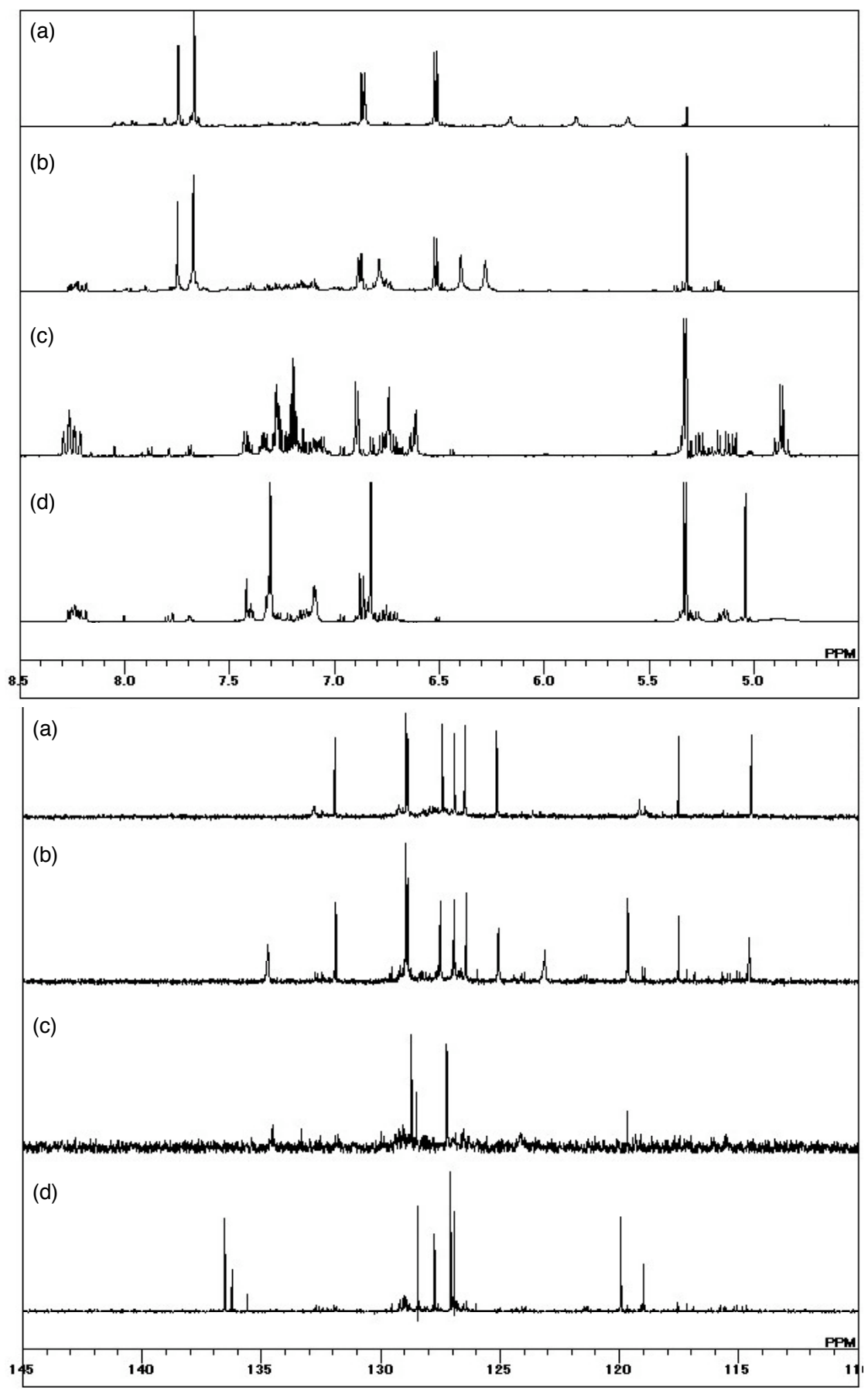

(a) Zr catalyst prepared in situ. (b) Isolated Zr catalyst powder. (c) Single crystals of Zr complex. (d) Single crystals of Zr complex with NMI. 
Imine and catalyst (Figure S-4). $\quad$ (a) 1-Naphthaldimine 3 (10 mg) was dissolved in dichloromethane- $d^{2}(0.50 \mathrm{~mL})$. (b) $N$-Methylimidazole $(0.48 \mathrm{mmol})$ was added to $(R)$-6,6'-dibromoBINOL $(0.08 \mathrm{mmol})$ and $\mathrm{Zr}\left(\mathrm{O}^{\mathrm{i}} \mathrm{Pr}\right)_{4}(0.04 \mathrm{mmol})$ in dichloromethane- $d^{2}$ $(0.50 \mathrm{~mL})$ at room temperature. The mixture was stirred for $1 \mathrm{~h}$ at the same temperature, and then $3(9.9 \mathrm{mg}, 0.04 \mathrm{mmol})$ was added to the mixture. (c) The catalyst $(23 \mathrm{mg}, 0.02 \mathrm{mmol})$ and $3(5.0 \mathrm{mg}, 0.02 \mathrm{mmol})$ were dissolved in dichloromethane- $d^{2}(0.50 \mathrm{~mL})$. (d) The crystal $(10 \mathrm{mg}, 3.2 \mu \mathrm{mol})$ and naphthaldimine (3.2 $\mathrm{mg}, 0.013 \mathrm{mmol})$ were dissolved in dichloromethane- $d^{2}(0.50 \mathrm{~mL})$. (e) $N$-Methylimidazole $(2.1 \mu \mathrm{L}, 8$ equiv to $\mathrm{Zr}$ ) was added to white suspension in dichloromethane- $d^{2}(0.50 \mathrm{~mL})$ containing the crystal $(10 \mathrm{mg}, 3.2 \mu \mathrm{mol})$. After stirred for $3 \mathrm{~h}$, crystals were completely dissolved and then $3(3.2 \mathrm{mg}, 0.013 \mathrm{mmol})$ was added to the solution.

Figure S-4. ${ }^{1} \mathrm{H}$ NMR spectra of imine 3 and $\mathrm{Zr}$ catalysts in $\mathrm{CD}_{2} \mathrm{Cl}_{2}$.

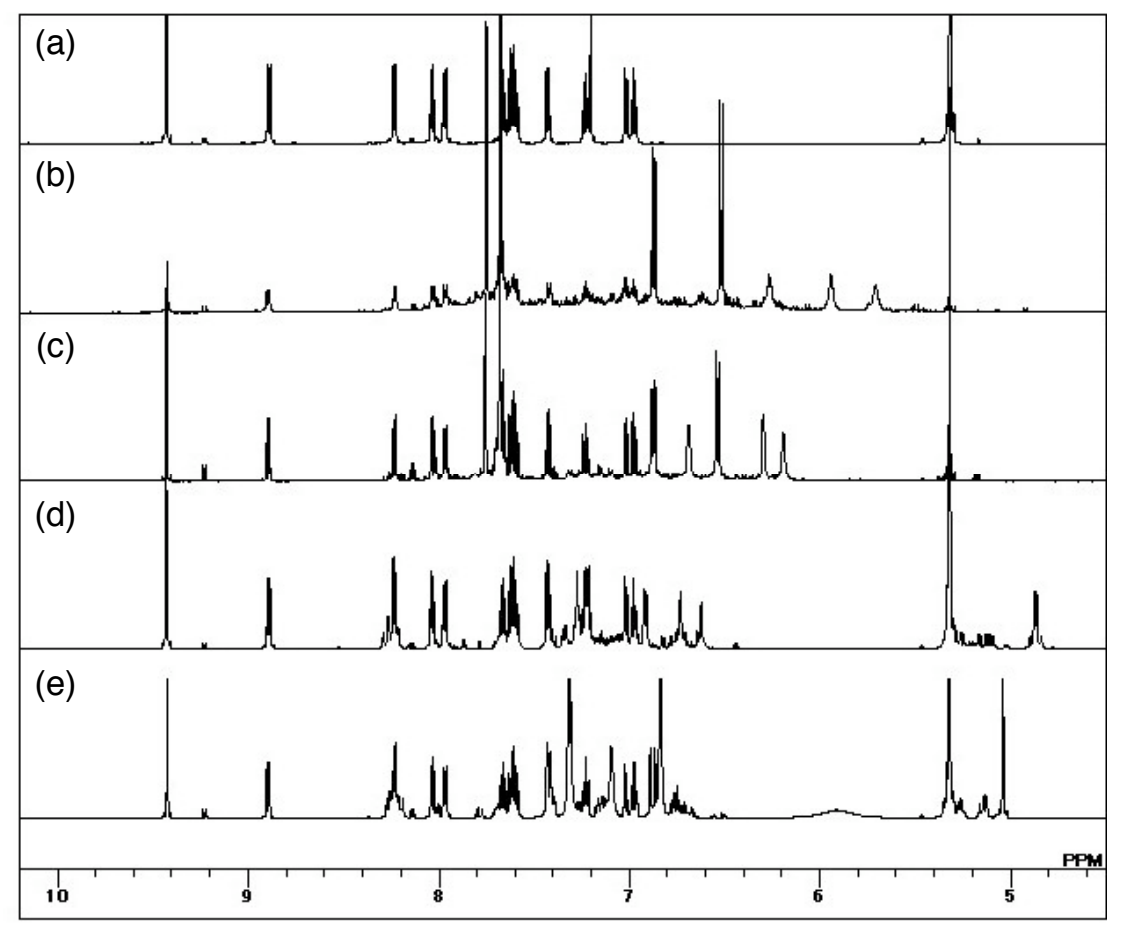

(a) Imine 3. (b) Imine 3 and Zr catalyst prepared in situ. (c) Imine 3 and isolated $\mathrm{Zr}$ catalyst powder. (d) Imine 3 and single crystals of $\mathrm{Zr}$ complex. (e) Imine 3 and single crystal of $\mathrm{Zr}$ complex with NMI. 


\section{References and Notes}

(1) Astudillo, H. E. A.; Chokotho, N. C. J.; Jarvis, T. C.; Johnson, C. D.; Lewis, C. C.; Mcdonnell, P. D. Tetrahedron 1985, 41, 5919.

(2) (a) Kobayashi, S.; Manabe, K.; Ishitani, H.; Matsuo, J. "Silyl Enol Ethers", in Science of Synthesis, Houben-Weyl Methods of Molecular Transformations; Bellus, D.; Ley, S. V.; Noyori, R.; Regitz, M.; Schaumann, E.; Shinkai, I.; Thomas, E. J.; Trost, B. M., Eds.; Georg Thieme Verlag: Stuttgart, 2002; vol. 4, p. 317. (b) Ireland, R. E.; Wipf, P.; Armstrong III, J. D. J. Org. Chem. 1991, 56, 650. (c) Heathcock, C. H.; Buse, C. T.; Kleschick, W. A.; Pirrung, M. C.; Sohn, J. E.; Lampe, J. J. Org. Chem. 1980, 45, 1066.

(3) (a) Ishitani, H.; Ueno, M.; Kobayashi, S. J. Am. Chem. Soc. 1997, 119, 7153. (b) Ishitani, H.; Ueno, M.; Kobayashi, S. J. Am. Chem. Soc. 2000, 122, 8180. (c) Ueno, M.; Ishitani, H.; Kobayashi, S. Org. Lett. 2002, 4, 3395. 